\title{
Correlation of coronary artery stenosis evaluation with left heart structure and function by multi-slice computed tomography
}

\author{
L.N. Song, A.D. Cao, Y.J. Niu and N. Liu \\ Department of Radiology, \\ The First Affiliated Hospital of Liaoning Medical University, Jinzhou, China \\ Corresponding author: Y.J. Niu \\ E-mail: jhdoccn@126.com
}

Genet. Mol. Res. 13 (3): 5940-5948 (2014)

Received May 28, 2013

Accepted April 9, 2014

Published August 7, 2014

DOI http://dx.doi.org/10.4238/2014.August.7.9

\begin{abstract}
The aim of this study was to determine the impact of multi-slice computed tomography (MSCT) evaluation of coronary artery stenosis on left heart structure and systolic function. Coronary artery CT angiography was performed in 200 patients diagnosed with coronary heart disease, and then according to the AHA coronary artery 17-segment fractionation method, the Gensini score (GS) was determined for every narrow segment, and one-stop assessment of the correlation between left heart structure and function was performed. After the grouping of GS quartiles from low to high, there were differences between different patients with regard to LVDD, LADD, LVEDV, LVESV, MM, LVEF, and FS, while no difference in SV and CO. GS showed linear negative correlation with LVEF and FS, and linear positive correlation with LVDD, LADD, LVEDV, LVESV, and $\mathrm{MM}$, while no correlation with SV and CO. That is, GS of coronary artery stenosis was negatively correlated with left ventricular systolic function and positively correlated with myocardial mass. The narrower the coronary artery, the worse the cardiac function and the higher the myocardial hypertrophy. Coronary artery stenosis was one of the
\end{abstract}


important causes of the decrease in left ventricular systolic function and cardiac remodeling.

Key words: Tomography; X-ray computed tomography; Left ventricular systolic function; Coronary artery stenosis; Left ventricular ejection fraction

\section{INTRODUCTION}

Currently, cardiovascular disease has become "the number one killer", thereby a serious hazard to human health. Epidemiological investigation of coronary heart disease has shown a rising trend in morbidity and mortality year by year, and the changes in heart function directly affect the quality of life and survival rate of the patients with coronary heart disease. Studies have shown that accurate determination of left ventricular function is an important basis for clinical diagnosis, risk stratification, therapy planning, and prognosis evaluation (Zhang et al., 2009; Ito et al., 2010).

In the fields of investigating the relationship between the degree of coronary artery disease and heart function, foreign and domestic scholars often use traumatic selective coronary angiography to determine the degree of stenosis with visual observation, and then compare with transthoracic echocardiography evaluation results of cardiac function, in which collecting adequate data is difficult. Furthermore, there is often a certain time interval between the two examinations, so the cardiac function would not correspond exactly to the degree of stenosis at the time of selective coronary angiography, and transthoracic echocardiography would be affected by artificial influence. Multi-slice computed tomography (MSCT) has shown an advantage in the diagnosis of coronary arterial stenosis and cardiac function. With the continuous improvement of time and spatial resolution in MSCT, multi-slice spiral CT coronary artery angiography (MSCTCA) could be used for the evaluation of coronary arterial stenosis, and the original data could also be used for a one-stop cardiac function determination. Studies have shown that 64-layer SCTCA has high sensitivity and specificity in the diagnosis of coronary artery stenosis, and its results for cardiac function parameters were found to be highly correlated with the gold standard cMRI (Annuar et al., 2008; Groen et al., 2009; Zhang et al., 2009; Ito et al., 2010). In the present study, 128-layer SCTCA was used, along with retrospective ECG gating techniques (Mollet et al., 2005; Lu et al., 2008), to continuously scan several cardiac cycles, and the real-time dynamic 4-D raw image data of the systolic and diastolic ends, namely the "time-space" periods, were analyzed for the one-stop noninvasive evaluation of coronary artery stenosis, left ventricular structure and systolic function (Ko et al., 2010; Takx et al., 2012). Accordingly, we explored the relationship of degree of coronary artery stenosis and left ventricular systolic function and ventricular remodeling, providing basic data for clinical diagnosis, risk stratification, efficacy evaluation, and prognosis follow-up, which would especially be of great significance for the prevention of congestive heart failure.

\section{MATERIAL AND METHODS}

\section{Subjects}

Two hundred patients were collected from the outpatient and inpatient clinics in our 
hospital, and enrolled in the study from January to December 2012. They were 38 to 88 years old, with an average age of $58 \pm 23$ years, including 130 males and 70 females, who all underwent MSCTCA. This study was conducted in accordance with the Declaration of Helsinki and with approval from the Ethics Committee of Liaoning Medical University. Written informed consent was obtained from all participants.

\section{Inclusion criteria}

Suspected or diagnosed patients were included if they had no history of iodine allergy or coronary artery bypass grafting and percutaneous coronary intervention, no tachyarrhythmia and ectopic pacemaker rhythm, no valvular heart disease, no congenital heart disease, and no hypertensive heart disease or myocardial disease; pregnant women and patients with liver and kidney dysfunction were excluded.

\section{Scan methods and parameters}

Scanning method: Definition AS (64-detector 128-slice CT; Siemens, Munich, Germany) was first performed to obtain the calcification score, and a high-pressure syringe was then used for the injection of 65-70 mL non-ionic contrast medium iohexol $(350 \mathrm{mg} / \mathrm{mL}$; Beijing Beilu Pharmaceutical Co., Ltd., China) via the antecubital vein, at a flow rate of 3.5 to 5.0 $\mathrm{mL} / \mathrm{s}$, with an additional $40 \mathrm{~mL}$ physiological saline (to reduce the amount of contrast agent and the artifacts caused by the exceedingly high contrast agent concentrations in the superior vena cava and right atrium). The aortic root region was set as the region of interest, triggering threshold as $100 \mathrm{HU}$, then performed the automatical-tracking timing bolus of contrast agent, measuring the time-density curve to calculate the scan delay time, then performed the coronary angiography scan, scanned from the trachea carina to the heart diaphragmatic surface under the control of retrospective ECG gating; the scanning time was set as about 6 to $11 \mathrm{~s}$. Scan parameters were as follows: voltage, $120 \mathrm{kV}$; current, automatic mA; field of vision, 160 to $220 \mathrm{~mm}$; pitch, 0.2 to 0.5 , automatically selected with heart rate scanner; detector basic value, $64 \times 0.6 \mathrm{~mm}$, slice thickness, $0.6 \mathrm{~mm}$, speed, $0.33 \mathrm{~s} / \mathrm{r}$; matrix, 512 × 512; and convolution kernel, B26F. Heart rate was controlled within $75 \mathrm{bpm}$, where the patients whose heart rate was more than $75 \mathrm{bpm}$ were given 25 to $50 \mathrm{mg}$ Betaloc sublingually $30 \mathrm{~min}$ before the examination to lower the heart rate.

\section{Image postprocessing and measurement}

The circulation software was used to reconstruct 10 sets of images of the $10 \% \mathrm{RR}$ reformation interval according to the raw 0 to $90 \%$ RR interval data of ECG reformation interval, and 4-D preview mode was then used to determine the diastolic and systolic ends. The diastolic image was postprocessed, including multi-planar reformation, curved planar reformation, volume rendering technique, maximum intensity projection, and digital subtraction angiography flipping for coronary artery evaluation, as shown in Figure 1A and B. Measured horizontally were the left ventricular end-diastolic dimension (LVDD), left ventricular end-systolic dimension (LVSD) and the left atrium end-diastolic dimension (LADD) through the chordae tendineae of the mitral valve. The left ventricular shortening fraction $(\mathrm{FS})=(\mathrm{LVDD}-\mathrm{LVSD}) /$ 
LVDD (Figure 1C and D). The left ventricular systolic function was measured by determining the septal position, and then allowing the software to automatically draw the outline of the left ventricular inner and outer membranes. After manual correction, the software automatically calculated the left ventricular ejection fraction (LVEF), left ventricular end-diastolic volume (LVEDV), left ventricular end-systolic volume (LVESV), stroke volume (SV), cardiac output (CO), and myocardial mass (MM), as shown in Figure 2.
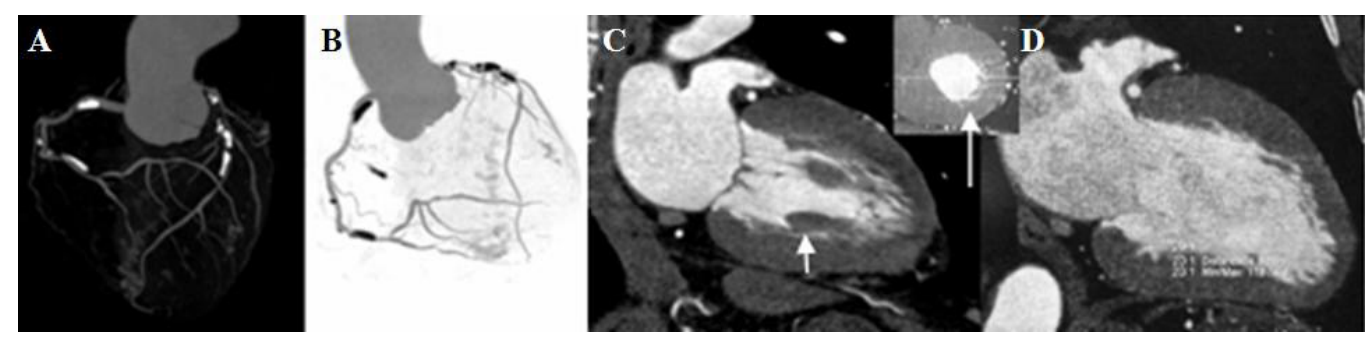

Figure 1. Coronary artery calcification imaging and the calculation method of left ventricle fraction shortening (FS). A. Maximum intensity projection image; B. Coronary calcification in Angioview DSA inversion image; C. Multi-planar reformation (MPR) image of systolic length; D. MPR section of diastolic length. The white short arrow refers to papillary muscle, and the long arrow refers to the horizontal section of mitral valve in systole.

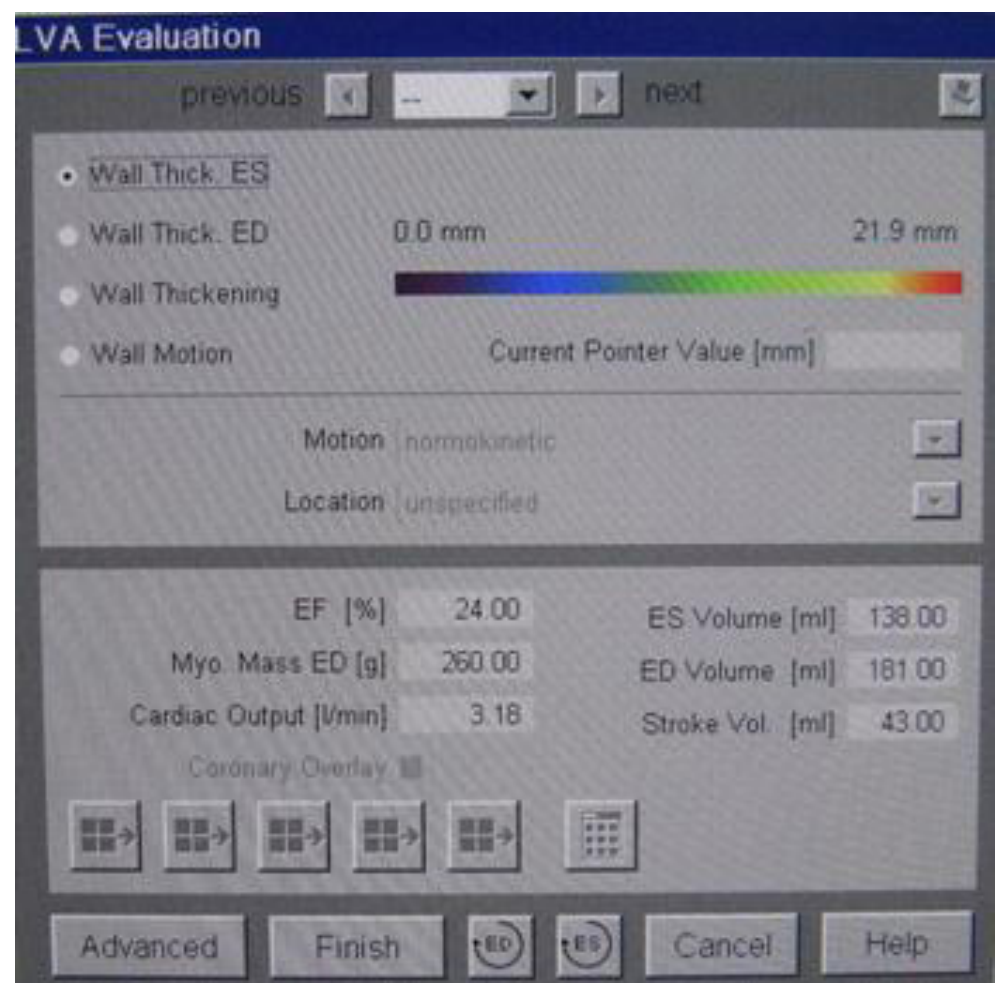

Figure 2. Analytical results of heart function with the Circulation software. Circulation cardiac function analysis software results: $\mathrm{LVEF}=24.00 \%, \mathrm{MM}=260.00 \mathrm{~g}, \mathrm{EDV}=181.00 \mathrm{~mL}, \mathrm{SV}=138.00 \mathrm{~mL}, \mathrm{SV}=43.00 \mathrm{~mL}, \mathrm{CO}$ $3.18 \mathrm{~L} / \mathrm{min}$. 


\section{Coronary artery evaluation method}

According to the improved coronary segmentation method of the American Heart Association (AHA) (Gensini, 1983; Bruners et al., 2009), the coronary artery tree was divided into 17 segments, and the Circulation Vascular Analysis software was used for image processing and analysis. In this experiment, according to the principle of the visual method, the cross-sectional area was used for the stenosis assessment, i.e., the degree of stenosis $=[$ (normal vascular area adjacent to the stenosis - area of the stenosis) / normal vascular area adjacent to the stenosis] (Figure 3), and the modified Gensini score (GS) system (Annuar et al., 2008) was used for the quantitative scoring of the degrees of vascular lesions, recorded as GS. Different degrees of coronary artery stenosis, namely 25, 50, 75, 90, 99 and 100\%, were rated 1, 2, 4, 8, 16, and 32 points, and different coronary segments were multiplied by the appropriate factors: pathological changes on the left main coronary artery, score x 5 ; left anterior descending artery, proximal segment, score x 2.5 ; left anterior descending artery, middle segment, score x 1.5, distal segment, score x 1; the first diagonal branch, score x 1 ; the second diagonal branch, score $\mathrm{x} 0.5$; left circumflex artery, proximal segment, score x 2.5; posterior descending and distal segment, score $\mathrm{x} 1$; posterior branch, score $\mathrm{x} 0.5$; the right coronary, proximal, middle, distal, and posterior descending segment, score $\mathrm{x} 1$. The final points were the summary of the branch points.

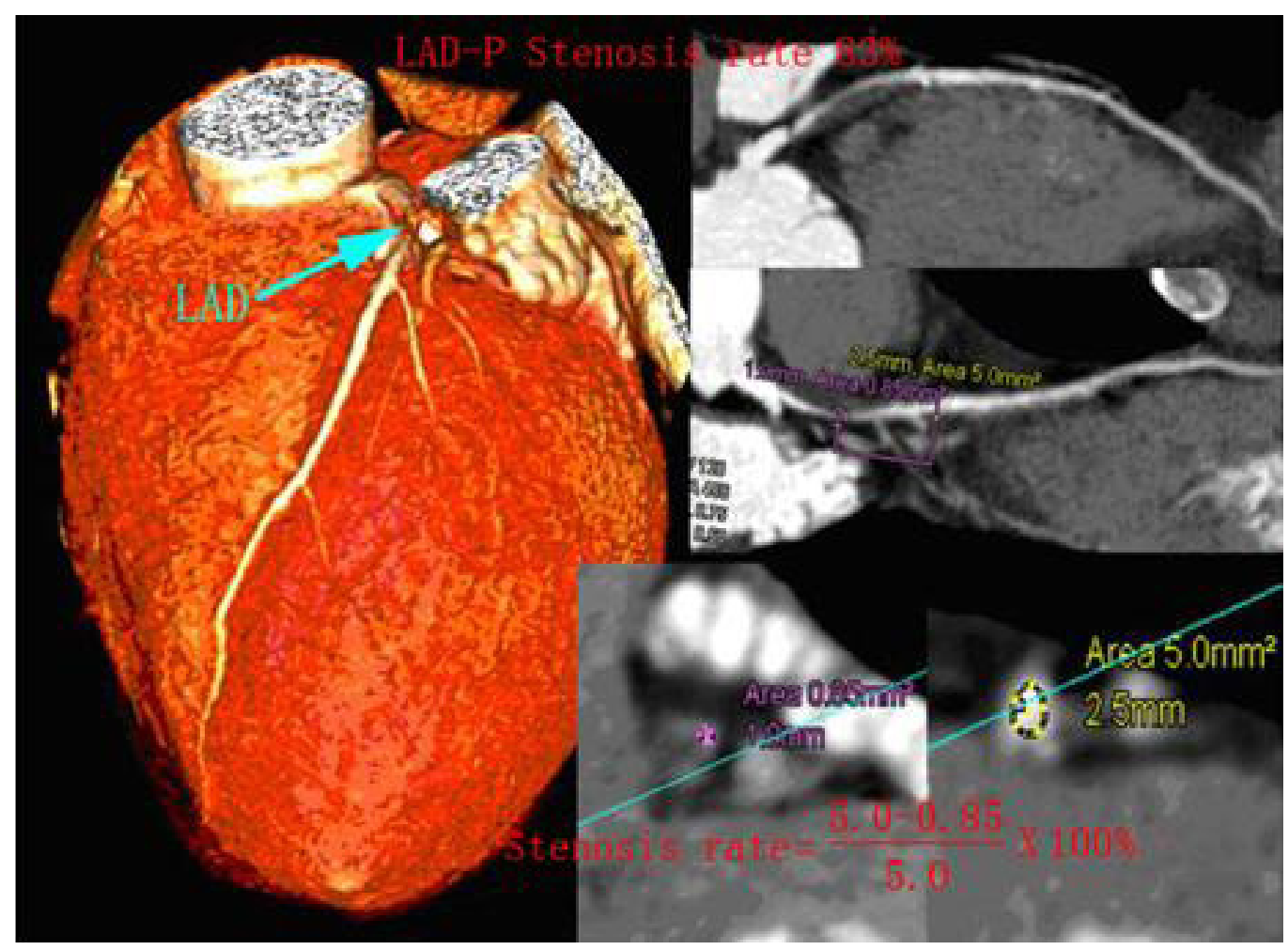

Figure 3. Calculation of the proximal stenosis degree of left anterior descending branch (LAD). Section area method of coronary stenosis (the same patient as in Figure 2), VR and CPR images: LAD proximal stenosis degree $=\left(5.0 \mathrm{~mm}^{2}-0.85 \mathrm{~mm}^{2}\right) \times 100 \% /\left(5.0 \mathrm{~mm}^{2}\right)=83 \%$. 


\section{Statistical analysis}

The SPSS17.0 software was used for the statistical processing. Measurement data are reported as means \pm standard deviation. Intragroup comparison was performed by singlefactor analysis of variance (ANOVA); the correlation between GS and cardiac function indices was assessed using the Spearman test.

\section{RESULTS}

\section{General information}

All 200 patients successfully completed the examination, without allergy to the contrast agent and leakage phenomenon, and the image quality was excellent and met the demands of coronary stenosis analysis and the quantitative evaluation of left ventricular structure and function indicators. The average post-inspiratory breath-hold time was $15 \pm 7 \mathrm{~s}$, and 54 patients took metoprolol at a dose of 12.5 to $50 \mathrm{mg}$. The average heart rate was $64.5 \pm 7.3$ bpm (50 to $85 \mathrm{bpm})$.

\section{Coronary artery GS and the left heart structure and function}

Having grouped the patients into quartile groups from low to high according to the GS of the degree of coronary artery stenosis, the means of LVDD, LADD, LVEDV, LVESV, MM, LVEF, and FS showed statistically significant differences between the different groups (all $\mathrm{P}<$ 0.05 ), indicating that with increasing GS of coronary artery disease, LVDD, LADD, LVEDV, and LVESV increased to various degrees, while LVEF and FS decreased to different degrees. That is, the more severe the coronary stenosis, or the higher the GS, the worse heart function is; SV and CO showed no statistically significant differences between the groups (all $\mathrm{P}>0.05$ ), which might have been related to the compensatory role of the FS law-regulating mechanisms (Table 1).

Table 1. Comparison of left ventricular structure, function and coronary Gensini score (GS).
\begin{tabular}{lcccrrr}
\hline Parameters & $\mathrm{GS}<5(\mathrm{~N}=90)$ & $5 \leq \mathrm{GS}<25(\mathrm{~N}=60)$ & $25 \leq \mathrm{GS}<60(\mathrm{~N}=40)$ & $\mathrm{GS} \geq 61(\mathrm{~N}=10)$ & $\mathrm{F}$ & $\mathrm{P}$ \\
\hline LVDD $(\mathrm{mm})$ & $4.45 \pm 0.56$ & $4.79 \pm 0.49$ & $5.69 \pm 0.69$ & $6.83 \pm 0.77$ & 7.183 & 0.001 \\
LADD $(\mathrm{mm})$ & $3.43 \pm 0.47$ & $3.69 \pm 0.45$ & $3.71 \pm 0.58$ & $3.83 \pm 0.52$ & 7.973 & 0.001 \\
LVEDV $(\mathrm{mL})$ & $102.18 \pm 29.28$ & $113.37 \pm 23.01$ & $168.67 \pm 22.17$ & $199.35 \pm 39.16$ & 7.052 & 0.001 \\
LVESV $(\mathrm{mL})$ & $39.57 \pm 15.62$ & $53.90 \pm 18.59$ & $119.26 \pm 26.77$ & $161.33 \pm 23.42$ & 13.512 & $<0.000$ \\
SV $(\mathrm{mL})$ & $62.53 \pm 20.31$ & $59.30 \pm 21.30$ & $49.67 \pm 7.08$ & $48.91 \pm 11.88$ & 1.865 & 0.159 \\
CO (L/min) & $3.69 \pm 1.07$ & $3.60 \pm 1.50$ & $3.23 \pm 1.17$ & $3.05 \pm 1.16$ & 0.370 & 0.776 \\
MM (g) & $138.21 \pm 21.28$ & $187.90 \pm 24.12$ & $208.33 \pm 33.20$ & $221.07 \pm 37.24$ & 9.493 & $<0.000$ \\
LVEF $(\%)$ & $61.41 \pm 8.72$ & $51.6 \pm 10.50$ & $35.50 \pm 9.91$ & $31.66 \pm 11.59$ & 6.841 & 0.001 \\
FS (\%) & $36.05 \pm 2.82$ & $35.45 \pm 5.38$ & $35.69 \pm 4.68$ & $31.07 \pm 6.75$ & 8.592 & 0.001 \\
\hline Data & & &
\end{tabular}

Data are reported as means $\pm \mathrm{SD}$.

\section{Correlation analysis}

GS showed negative correlation with LVEF and FS $(\mathrm{P}<0.05)$, indicating that the LVEF and FS values decreased, while the internal diameters of the left atrium and ventricle increased; GS was positively correlated with LVDD, LADD, LVEDV, and LVESV in a linear fashion $(\mathrm{P}<$ 
$0.05)$, indicating that the greater the coronary artery stenosis, the higher the GS, LVDD, LADD, LVEDV, and LVESV were accordingly; GS showed no significant correlation with CO and SV ( $\mathrm{P}>0.05$ ), which might have been correlated with the compensatory role caused by the FS adjustment mechanism; GS and MM were linearly and positively correlated, and MM was negatively correlated with LVEF and FS $(r=-0.596,-0.732)(P<0.05)$, suggesting that the higher the GS, the much more severe MM is, and the worse systolic function is (Table 2).

\begin{tabular}{|c|c|c|c|c|c|c|c|c|c|}
\hline \multirow[t]{2}{*}{ Parameters } & \multicolumn{5}{|c|}{ Structural parameters of left heart } & \multicolumn{4}{|c|}{ Functional parameters of left heart } \\
\hline & LVDD (mm) & LADD (mm) & LVEDV (mL) & LVESV (mL) & $\mathrm{SV}(\mathrm{mL})$ & $\mathrm{CO}(\mathrm{L} / \mathrm{min})$ & MM (g) & LVEF (\%) & FS (\%) \\
\hline $\mathrm{r}$ & 0.595 & 0.503 & 0.567 & 0.690 & -0.283 & -0.183 & 0.618 & -0.575 & -0.490 \\
\hline $\mathrm{P}$ & 0.001 & 0.028 & 0.002 & 0.000 & 0.009 & 0.327 & 0.006 & 0.001 & 0.007 \\
\hline
\end{tabular}

\section{DISCUSSION}

Coronary artery stenosis can cause angina and myocardial infarction, further leading to the failure of myocardial contractile function, cardiac enlargement and lower compliance. Coronary angiography is the "gold standard" for the diagnosis of coronary heart disease, but because it is an invasive examination, it is often not easily accepted by the patient. The application of MSCT, accompanied by retrospective ECG gating technology, could evaluate cardiac function and coronary artery stenosis noninvasively, and with the combination of MSCTCA-determining coronary artery stenosis, the relationship between coronary artery stenosis and cardiac morphology and function was considered in this study.

According to the degree of coronary artery stenosis or GS grouping, the results showed that with increase in coronary artery score, there was an increase in LVDD, LADD, LVEDV, and LVESV to various degrees, while LVEF and FS decreased to different degrees, consistent with that reported in the literature (Artmann et al., 2009; Mahnken et al., 2009; Xue et al., 2012). The Spearman correlation analysis of the GS results demonstrated that GS was negatively and significantly correlated with LVEF and FS, positively and significantly correlated with LVDD, LADD, LVEDV, and LVESV, with no significant correlation in regard to SV and CO. This indicated that the higher the GS, the higher the LVDD, LADD, LVEDV, and LVESV and the lower the LVEF and FS were, suggesting an increase in the inner diameter of left atrium and ventricle, which could be used to predict the severity of coronary artery disease. The possible mechanism may be like the following: myocardial ischemia would lead to a decrease in myocardial contractility, the left ventricle and left atrium would then congest, and the left atrial systolic diameter and diastolic diameter would then expand, leading to left ventricular remodeling, which would feedback and increase left ventricular systolic function failure. Thus, the severer the coronary stenosis is, the higher the GS is, which means the worse heart function. When the coronary artery diameter stenosis is less than $75 \%$, stenosis has less effect on ventricular systolic function, and the stenosis developed more obviously, it would further reduce systolic dysfunction. When the extent of coronary stenosis is more than $90 \%$, LVEF significantly decreases (Ashrafian et al., 2008; D'Ancona et al., 2008; Wang et al., 2009b). The fact that GS was not statistically associated with SV and CO may be connected with the Frank-Starling mechanism (Leber et al., 2005; Ashrafian et al., 2008; D'Ancona et al., 2008). That is, when a coronary artery stenosis causes moderate systolic heart failure, myocardial 
end-diastolic fiber length increases by the FS law-regulating mechanism, and the ventricular end-diastolic volume (ventricular preload) increases, the resting $\mathrm{CO}$ and ventricular function could maintain at normal levels.

The cardiac muscle is the driving force of the systole, where changes in the MM would be bound to cause changes in myocardial contractility, thereby affecting cardiac output. Therefore, MM could be used to express ventricular function. The results of this study showed that GS and MM had a good positive correlation and that MM had a good negative correlation with LVEF, suggesting that the higher the GS is, the severer the MM is, which leads to worse systolic function. Studies have shown that MM is a strong predictor of sudden cardiac death and that MM has a very close relationship with cardiac function, where it is one of the most sensitive indicators of cardiac function (Nakamura, 2010; Li et al., 2012). Coronary artery stenosis is an important cause of the reduction of left ventricular systolic function and cardiac remodeling. It could cause heart expansion (left atrium and left ventricle), mainly due to myocardial ischemia, myocardial cell edema, interstitial hyperplasia and left ventricular remodeling, resulting in a decrease in left ventricular compliance and cardiac function. As the degree of stenosis increases, MM will increase, suggesting that left ventricular MM could be used as an effective indicator to determine left ventricular dysfunction, especially valuable for those patients with normal early LVEF values and no effects on general activities, and in whom MM is relatively stable in a certain period, with strong repeatability (Chander et al., 2008; Wang et al., 2009a; Krim et al., 2011).

In this research, 128-layer SCT, accompanied by retrospective ECG-gating technology, was used to evaluate cardiac function, and noninvasively assess the relationship of coronary artery stenosis (morphology) and cardiac function (functional study). This showed great advantages: the scanning speed was fast, easy, safe, noninvasive, and of low cost; synchronism was good, in which image data of the coronary artery could be obtained and cardiac function determined in one-stop access, without examination interval, where cardiac function could reflect the current vein stenosis. We also found that there were some deficiencies: the time and spatial resolutions needed to be further improved, and there were still partial motion artifacts, which could affect the precise definition of left ventricular systolic end and diastolic end; and breathing exercises, rapid heart rate, heart rate arrhythmia, and severe coronary calcification would all affect coronary stenosis assessment (Ruixing et al., 2007; Chander et al., 2008). In addition, whether the use of metoprolol and contrast agents and saline in the contrast could affect left ventricular function still remains to be further studied.

\section{ACKNOWLEDGMENTS}

Research supported by the Science and Technology Plan Project of LiaoNing Province, China (\#2013225305).

\section{REFERENCES}

Annuar BR, Liew CK, Chin SP, Ong TK, et al. (2008). Assessment of global and regional left ventricular function using 64-slice multislice computed tomography and 2D echocardiography: a comparison with cardiac magnetic resonance. Eur. J. Radiol. 65: 112-119.

Artmann A, Enayati S, Ratzenbock M, Schwarz M, et al. (2009). Image quality of CT angiography of coronary arteries depending on the degree of coronary calcification using a dual source CT scanner. Rofo 181: 863-869. 
Ashrafian H, Williams L and Frenneaux MP (2008). The pathophysiology of heart failure: a tale of two old paradigms revisited. Clin. Med. 8: 192-197.

Bruners P, Knackstedt C, Mahnken AH, Luhmann N, et al. (2009). Global left ventricular function: assessment with dualsource CT versus conventional ventriculography in a porcine model. Acta Cardiol. 64: 311-319.

Chander A, Brenner M, Lautamaki R, Voicu C, et al. (2008). Comparison of measures of left ventricular function from electrocardiographically gated $82 \mathrm{Rb}$ PET with contrast-enhanced CT ventriculography: a hybrid PET/CT analysis. J. Nucl. Med. 49: 1643-1650.

D'Ancona G, Biondo D, Mamone G and Marrone G (2008). Ischemic mitral valve regurgitation in patients with depressed ventricular function: cardiac geometrical and myocardial perfusion evaluation with magnetic resonance imaging. Eur. J. Cardiothorac. Surg. 4: 964-968.

Gensini GG (1983). A more meaningful scoring system for determining the severity of coronary heart disease. Am. $J$. Cardiol. 51: 606.

Groen JM, van der Vleuten PA, Greuter MJ, Zijlstra F, et al. (2009). Comparison of MRI, 64-slice MDCT and DSCT in assessing functional cardiac parameters of a moving heart phantom. Eur. Radiol. 19: 577-583.

Ito Y, Ito K, Shiroto T, Tsuburaya R, et al. (2010). Cardiac shock wave therapy ameliorates left ventricular remodeling after myocardial ischemia-reperfusion injury in pigs in vivo. Coron. Artery Dis. 21: 304-311.

Ko SM, Kim YJ, Park JH and Choi NM (2010). Assessment of left ventricular ejection fraction and regional wall motion with 64-slice multidetector CT: a comparison with two-dimensional transthoracic echocardiography. Br. J. Radiol. 83: 28-34.

Krim SR, Jiang AF, Vivo RP, Little SH, et al. (2011). Gigantic coronary sinus associated with concurrent persistent left superior vena cava and right ventricular volume overload. Methodist. Debakey. Cardiovasc. J. 6: 46-47.

Leber AW, Knez A, von Ziegler F, Becker A, et al. (2005). Quantification of obstructive and nonobstructive coronary lesions by 64-slice computed tomography: a comparative study with quantitative coronary angiography and intravascular ultrasound. J. Am. Coll. Cardiol. 46: 147-154.

Li JM, Shi RF, Zhang LR and Li T (2012). Application of CT angiography in diagnosing functional relevant coronary stenoses. Chin. J. Med. Imag. 20: 703-706.

Lu J, Wang LS, Jia EZ and Yong YH (2008). Study on the relationship between the stenosis of coronary and left ventricular cardiac function. J. Nanjing Med. Univ. (Natural Science Edition) 28: 1007-1010.

Mahnken AH, Bruners P, Schmidt B, Bornikoel C, et al. (2009). Left ventricular function can reliably be assessed from dual-source CT using ECG-gated tube current modulation. Invest. Radiol. 44: 384-389.

Mollet NR, Cademartiri F, van Mieghem CA, Runza G, et al. (2005). High-resolution spiral computed tomography coronary angiography in patients referred for diagnostic conventional coronary angiography. Circulation 112: 23182323.

Nakamura M (2010). Angiography is the gold standard and objective evidence of myocardial ischemia is mandatory if lesion severity is questionable. - Indication of PCI for angiographically significant coronary artery stenosis without objective evidence of myocardial ischemia (Pro)-. Circ. J. 75: 204-210.

Ruixing Y, Jinzhen W, Dezhai Y and Jiaquan L (2007). Cardioprotective role of cardiotrophin-1 gene transfer in a murine model of myocardial infarction. Growth Factors 25: 286-294.

Takx RA, Moscariello A, Schoepf UJ, Barraza JM Jr, et al. (2012). Quantification of left and right ventricular function and myocardial mass: comparison of low-radiation dose 2nd generation dual-source CT and cardiac MRI. Eur. $J$. Radiol. 81: e598-e604.

Wang S, Fei K, Xu YW, Wang LX, et al. (2009a). Dihydroxyflavonol reduces post-infarction left ventricular remodeling by preventing myocyte apoptosis in the non-infarcted zone in goats. Chin. Med.J. 122: 61-67.

Wang YT, Yang CY, Hsiao JK, Liu HM, et al. (2009b). The influence of reconstruction algorithm and heart rate on coronary artery image quality and stenosis detection at 64-detector cardiac CT. Korean J. Radiol. 10: 227-234.

Xue P, Zheng HW, Chen Y and Zhang SJ (2012). 64-MDCT examination of left ventricular function: phase analysis of left ventricular end-diastolic and end-systolic. Radiol. Practice 27: 632-634.

Zhang P, Li BY, Zhou XS and Zhang M (2009). Meta analysis of 64 slice spiral CT in the diagnosis of coronary artery stenosis. J. Clin. Radiol. 28: 50-53. 